\title{
Sobolev inequalities for the symmetric gradient in arbitrary domains
}

\author{
Andrea Cianchi \\ Dipartimento di Matematica e Informatica "U. Dini", Università di Firenze \\ Viale Morgagni 67/A, 50134 Firenze, Italy \\ e-mail: cianchi@unifi.it \\ Vladimir G. Maz'ya \\ Department of Mathematics, Linköping University, SE-581 83 Linköping, Sweden \\ and \\ RUDN University \\ 6 Miklukho-Maklay St, Moscow, 117198, Russia \\ e-mail:vladimir.mazya@liu.se
}

\begin{abstract}
A form of Sobolev inequalities for the symmetric gradient of vector-valued functions is proposed, which allows for arbitrary ground domains in $\mathbb{R}^{n}$. In the relevant inequalities, boundary regularity of domains is replaced with information on boundary traces of trial functions. The inequalities so obtained exhibit the same exponents as in classical inequalities for the full gradient of Sobolev functions, in regular domains. Furthermore, they involve constants independent of the geometry of the domain, and hence yield novel results yet for smooth domains. Our approach relies upon a pointwise estimate for the functions in question via a Riesz potential of their symmetric gradient and an unconventional potential depending on their boundary trace.
\end{abstract}

\section{Introduction}

Diverse mathematical models for physical phenomena, described by some vector-valued function $\mathbf{u}: \Omega \rightarrow \mathbb{R}^{n}$, depend on the derivatives of $\mathbf{u}$ just through the symmetric part $\mathcal{E} \mathbf{u}$ of its distributional gradient $\nabla \mathbf{u}$. Here, $\Omega$ is an open set in $\mathbb{R}^{n}, n \geq 2$, and

$$
\mathcal{E} \mathbf{u}=\frac{1}{2}\left(\nabla \mathbf{u}+(\nabla \mathbf{u})^{T}\right)
$$

where $(\nabla \mathbf{u})^{T}$ stands for the transpose matrix of $\nabla \mathbf{u}$. Instances in this connection are provided by the theory of non-Newtonian fluids, where $\mathbf{u}$ represents the velocity of a fluid [2, DL, 22, 28, 39, 40, 41], and the theories of plasticity and nonlinear elasticity, where $\mathbf{u}$ stands for the displacement of a body [28, 32, 51]. The pertaining mathematical models amount to partial differential equations or variational integrals involving $\mathcal{E} \mathbf{u}$. The regularity theory for this kind

Mathematics Subject Classifications: 46E35, 46E30.

Key words and phrases: Sobolev inequalities, symmetric gradient, irregular domains, Riesz potential, boundary traces, Lorentz spaces, Orlicz spaces. 
of problems, which is considerably less developed than the classical one, calls for the use of an ad hoc functional framework.

This is provided by Sobolev type spaces, defined in analogy with the standard Sobolev spaces, where the role of $\nabla \mathbf{u}$ is instead played by $\mathcal{E} \mathbf{u}$. In particular, the homogeneous space $E^{1, p}(\Omega)$ is defined, for $p \geq 1$, as

$$
E^{1, p}(\Omega)=\left\{\mathbf{u} \in L_{\mathrm{loc}}^{1}(\Omega):|\mathcal{E} \mathbf{u}| \in L^{p}(\Omega)\right\} .
$$

Its subspace $E_{0}^{1, p}(\Omega)$ is defined as the space of those functions that "vanish" on $\partial \Omega$, in the sense that their continuation by 0 outside $\Omega$ belongs to $E^{1, p}\left(\mathbb{R}^{n}\right)$. Replacing $L^{p}(\Omega)$ in the definition of $E^{1, p}(\Omega)$ by a more general Banach function space $X(\Omega)$ yields a Sobolev type space for the symmetric gradient that will be denoted by $E^{1} X(\Omega)$. Namely,

$$
E^{1} X(\Omega)=\left\{\mathbf{u} \in L_{\text {loc }}^{1}(\Omega):|\mathcal{E} \mathbf{u}| \in X(\Omega)\right\} .
$$

The subspace $E_{0}^{1} X(\Omega)$ can be defined accordingly. The need for these generalized spaces arises, for example, in problems governed by nonlinearities of non-necessarily power type. They appear, for example, in the Prandt-Eyring fluids [6, 24, 28], in models for plastic materials with logarithmic hardening [25, as well as in models for the behavior of fluids in certain liquid body armors [31, 48, 53] which are affected by exponential type nonlinearities.

Sobolev type inequalities for spaces built upon the symmetric gradient are crucial in the regularity theory in question. Sobolev inequalties for the space $E_{0}^{1, p}(\Omega)$ have exactly the same form as those for the standard Sobolev space $W_{0}^{1, p}(\Omega)$, in any open set $\Omega$ of finite Lebesgue measure $\mathcal{L}^{n}(\Omega)$. In particular, when $p \in(1, \infty)$, this is a consequence of the Korn inequality, which tells us that, if $\mathbf{u} \in E_{0}^{1, p}(\Omega)$, then $\mathbf{u}$ is in fact weakly differentiable in $\Omega$, and $|\nabla \mathbf{u}| \in L^{p}(\Omega)$. Moreover,

$$
\|\nabla \mathbf{u}\|_{L^{p}(\Omega)} \leq c\|\mathcal{E} \mathbf{u}\|_{L^{p}(\Omega)}
$$

for some constant $c=c\left(n, p, \mathcal{L}^{n}(\Omega)\right)$ and every function $\mathbf{u} \in E_{0}^{1, p}(\Omega)$ [26, 29, 30, 32, 34, 44, 45, 47]. By contrast, this result fails in the endpoint cases when either $p=1$ or $p=\infty$ [19, 35, 46. Still, Sobolev inequalities with the same target norms as for $W_{0}^{1,1}(\Omega)$ continue to hold in $E_{0}^{1,1}(\Omega)-$ see [50], and the recent advances [49, 52].

If the zero boundary condition is dropped, namely if functions from $E^{1, p}(\Omega)$ are considered, and $\Omega$ is connected, a counterpart of inequality 1.3 still holds, where the norm of $\nabla \mathbf{u}$ on the left-hand side has to be replaced by the $L^{p}$ distance of $\nabla \mathbf{u}$ from the space of skew-symmetric $n \times n$ matrices. However, the resultant inequality requires suitable regularity assumptions on the domain $\Omega$. Lipschitz domains, or even John domains support this kind of Korn inequality [21, 23]. In absence of a Korn type inequality, a reduction to the case of $W^{1, p}(\Omega)$ is not available, and a direct method for embeddings of $E^{1, p}(\Omega)$ is required. A technique based on potential estimates, reminiscent of the original proof by Sobolev, is developed in [8], and also applies to less regular domains.

In the present paper we offer a sort of Sobolev type inequalities, involving the symmetric gradient, that requires no regularity on the domain at all. The point of view that will be adopted is that, in a Sobolev inequality for $\mathcal{E} \mathbf{u}$, information on the integrability of the bounday trace of $\mathbf{u}$ can serve as a substitute for boundary regularity of the ground domain $\Omega$, an assumption which is critical even in the usual situation when the full gradient $\nabla \mathbf{u}$ is employed.

To be more specific, the inequalities that will be dealt with have the form

$$
\|\mathbf{u}\|_{Y(\Omega, \mu)} \leq c\left(\|\mathcal{E} \mathbf{u}\|_{X(\Omega)}+\|\mathbf{u}\|_{Z(\partial \Omega)}\right)
$$


where $\|\cdot\|_{X(\Omega)}$ is a Banach function norm on $\Omega$ with respect to Lebesgue measure, $\|\cdot\|_{Y(\Omega, \mu)}$ is a Banach function norm with respect to a possibly more general upper Alfhors regular measure $\mu,\|\cdot\|_{Z(\partial \Omega)}$ is a Banach function norm on $\partial \Omega$ with respect to the $(n-1)$-dimensional Hausdorff measure $\mathcal{H}^{n-1}$, and $c$ is a constant. Besides the arbitrariness of $\Omega$, a key feature of the inequalities to be established is the fact that the norms $\|\cdot\|_{X(\Omega)},\|\cdot\|_{Y(\Omega, \mu)}$ and $\|\cdot\|_{Z(\partial \Omega)}$ in inequality (1.4) are exactly the same as those appearing in a counterpart inequality on regular domains, with $\nabla \mathbf{u}$ in the place of $\mathcal{E} \mathbf{u}$. Another distinctive trait is that the constant $c$ is independent of the regularity of the domain $\Omega$. Thereby, under this respect, our conclusions are new even in the case of smooth domains.

As pointed out above, due to the lack of Korn type inequalities in irregular domains, inequalities like (1.4) cannot be derived via their counterparts for the full gradient

$$
\|\mathbf{u}\|_{Y(\Omega, \mu)} \leq c_{1}\|\nabla \mathbf{u}\|_{X(\Omega)}+c_{2}\|\mathbf{u}\|_{Z(\partial \Omega)},
$$

that have recently been established, together with higher-order versions, in [16]. Another obstacle for such a derivation is that, even in regular domains $\Omega$, a Korn type inequality need not hold if $L^{p}(\Omega)$, with $p \in(1, \infty)$, is replaced by a more general Banach function norm $X(\Omega)$. Let us notice that failure of the Korn inequality is not limited to the norms in $L^{1}(\Omega)$ and in $L^{\infty}(\Omega)[5$. We refer to [1, 4, 5, 17, 15, 21, 27] for positive results in the case when the latter is a norm in an Orlicz space. Let us incidentally mention that, in the special situation when $\mu=\mathcal{L}^{n}, X=L^{p}, Y=L^{q}$, and $Z=L^{r}$, with $1 \leq p<n, r \geq 1$ and $q=\min \left\{\frac{r n}{n-1}, \frac{n p}{n-p}\right\}$, inequality 1.5 was established in [42] via isoperimetric inequalities. The optimal constants $c_{1}$ and $c_{2}$ were also exhibited in that paper, for $p=1$, in the setting of scalar functions. Mass transportation techniques have been exploited in [37] to determine the optimal constants when $1<p<n$. Sharp constants in a parallel inequality, corresponding to the borderline case when $p=n$, can be found in [38].

Our approach to inequality (1.4) starts with a fundamental pointwise estimate for functions $\mathbf{u} \in E^{1,1}(\Omega)$, in terms of the Riesz potential of order 1 applied to $|\mathcal{E} \mathbf{u}|$, plus a nonstandard potential depending on the values of $\mathbf{u}$ over $\partial \Omega$. This estimate is close in the spirit to the method of Sobolev, and its proof is inspired by arguments from [8] and [16]. It turns the problem of the validity of inequality (1.4) into that of the boundedness of the apropos potential operators between the function spaces $X(\Omega)$ and $Y(\Omega, \mu)$, and $Z(\partial \Omega)$ and $Y(\Omega, \mu)$, respectively. The new problem can be faced with the help of a reduction principle, which rests upon an inequality, in rearrangement form, deduced from the fundamental pointwise inequality. This principle enables us to reduce the question of the boundedness of the relevant $n$-dimensional operators to the considerably simpler problem of the boundedness of one-dimensional Hardy type operators. With this tool at disposal, one can establish inequalities of the form (1.4) for various families of Banach funcion spaces $X, Y$ and $Z$. As examples, we present results for Lebesgue, Lorentz and Zygmund spaces, and also obtain analogues of classical inequalities, such as the YudovichPohozaev-Trudinger exponential inequality, as special instances in bordeline situations. A Rellich type compactness theorem is given as well.

\section{A pointwise estimate}

Our estimate for functions $\mathbf{u} \in E^{1,1}(\Omega)$ at a point $x \in \Omega$ involves, loosely speaking, the value of the trace of $\mathbf{u}$ at the first point on $\partial \Omega$ intercepted on each ray issued from $x$. Since neither regularity nor boundedness is a priori assumed on $\Omega$, traces of weakly differentiable functions on $\partial \Omega$ need not be defined. Our pointwise estimate an the other results of this paper will thus 
be stated for functions that are continuous in $\bar{\Omega}$. More precisely, we shall make use of the space

$$
C_{b}(\bar{\Omega})=\{\mathbf{u}: \mathbf{u} \text { is continuous in } \bar{\Omega} \text { and has bounded support }\} .
$$

Of course, the norm inequalities that will be established for functions in $E^{1} X(\Omega) \cap C_{b}(\bar{\Omega})$, for some Banach function space $X(\Omega)$, continue to hold for the closure of $C_{b}(\bar{\Omega})$ in $E^{1} X(\Omega)$. Such a closure is known to agree with $E^{1} X(\Omega)$ itself if, for instance, $X=L^{p}$, and $\mathbb{R}^{n} \backslash \bar{\Omega}$ satisfies the cone condition [51, Proposition 1.3, Chapter 1].

Let us begin our discussion with a few notations and preliminary properties. Given any open set $\Omega$ in $\mathbb{R}^{n}$, with $n \geq 2$, and any point $x \in \Omega$, we set

$$
\Omega_{x}=\{y \in \Omega:(1-t) x+t y \subset \Omega \text { for every } t \in(0,1)\},
$$

and

$$
(\partial \Omega)_{x}=\{y \in \partial \Omega:(1-t) x+t y \subset \Omega \text { for every } t \in(0,1)\} .
$$

They are the largest subset of $\Omega$ and $\partial \Omega$, respectively, which can be "seen" from $x$. It is easily verified that $\Omega_{x}$ is an open set. Furthermore, $(\partial \Omega)_{x}$ is a Borel set - see [16, Proposition 3.1]. Next, define the sets

$$
\left(\Omega \times \mathbb{S}^{n-1}\right)_{0}=\left\{(x, \vartheta) \in \Omega \times \mathbb{S}^{n-1}: x+t \vartheta \in \partial \Omega \text { for some } t>0\right\},
$$

and

$$
\left(\Omega \times \mathbb{S}^{n-1}\right)_{\infty}=\left(\Omega \times \mathbb{S}^{n-1}\right) \backslash\left(\Omega \times \mathbb{S}^{n-1}\right)_{0},
$$

where $\mathbb{S}^{n-1}$ denotes the $(n-1)$-dimensional unit sphere in $\mathbb{R}^{n}$. Clearly,

$$
\left(\Omega \times \mathbb{S}^{n-1}\right)_{0}=\Omega \times \mathbb{S}^{n-1} \text { if } \Omega \text { is bounded } .
$$

Let

$$
\zeta:\left(\Omega \times \mathbb{S}^{n-1}\right)_{0} \rightarrow \mathbb{R}^{n}
$$

be the function defined as

$$
\zeta(x, \vartheta)=x+t \vartheta, \quad \text { where } t \text { is such that } x+t \vartheta \in(\partial \Omega)_{x} .
$$

Given a function $g: \partial \Omega \rightarrow \mathbb{R}^{m}, m \geq 1$, with compact support, we adopt the convention that $g(\zeta(x, \vartheta))$ is defined for every $(x, \vartheta) \in \Omega \times \mathbb{S}^{n-1}$, on extending it by 0 on $\left(\Omega \times \mathbb{S}^{n-1}\right)_{\infty}$; namely, we set

$$
g(\zeta(x, \vartheta))=0 \quad \text { if }(x, \vartheta) \in\left(\Omega \times \mathbb{S}^{n-1}\right)_{\infty} .
$$

Finaly, let us introduce the function

$$
\mathfrak{b}: \Omega \times \mathbb{S}^{n-1} \rightarrow(0, \infty]
$$

given by

$$
\mathfrak{b}(x, \vartheta)= \begin{cases}|\zeta(x, \vartheta)-x| & \text { if }(x, \vartheta) \in\left(\Omega \times \mathbb{S}^{n-1}\right)_{0}, \\ \infty & \text { otherwise. }\end{cases}
$$

One has that both $\zeta$ and $\mathfrak{b}$ are Borel functions [16, Proposition 3.2].

We are now in a position to state our pointwise bound. 
Theorem 2.1 [Pointwise estimate] Let $\Omega$ be any open set in $\mathbb{R}^{n}, n \geq 2$. There exists a constant $C=C(n)$ such that

$$
|\mathbf{u}(x)| \leq C \int_{\mathbb{S}^{n-1}}|\mathbf{u}(\zeta(x, \vartheta))| d \mathcal{H}^{n-1}(\vartheta)+C \int_{\Omega} \frac{|\mathcal{E} \mathbf{u}(y)|}{|x-y|^{n-1}} d y \quad \text { for } x \in \Omega
$$

for every function $\mathbf{u} \in E^{1,1}(\Omega) \cap C_{b}(\bar{\Omega})$. Here, convention (2.7) is adopted.

Remark 2.2 Under the assumption that

$$
u=0 \quad \text { on } \partial \Omega \text {, }
$$

inequality 2.10 reduces to

$$
|u(x)| \leq C \int_{\Omega} \frac{|\mathcal{E} \mathbf{u}(y)|}{|x-y|^{n-1}} d y \quad \text { for a.e. } x \in \Omega
$$

Proof of Theorem 2.1. Fix $x \in \Omega$. Given $\vartheta \in \mathbb{S}^{n-1}$, consider the function

$$
[0, \mathfrak{b}(x, \vartheta)] \ni t \mapsto \mathbf{u}(x+t \vartheta) \cdot \vartheta
$$

We claim that, for $\mathcal{H}^{n-1}$-a.e. $\vartheta \in \mathbb{S}^{n-1}$, this function is locally absolutely continuous, and

$$
\frac{d}{d t} \mathbf{u}(x+t \vartheta) \cdot \vartheta=\mathcal{E} \mathbf{u}(x+t \vartheta) \vartheta \cdot \vartheta \quad \text { for a.e. } t \in[0, \mathfrak{b}(x, \vartheta)]
$$

Note that this claim does not follow from standard properties of Sobolev functions, since $\mathbf{u}$ need not belong to $W_{\text {loc }}^{1,1}(\Omega)$, due to the failure of the Korn inequality for the $L^{1}$-norm. In order to prove it, let us begin by observing that, if $u$ is a smooth function, and we set $\mathbf{u}=\left(u^{1}, \ldots, u^{n}\right)$ and $\vartheta=\left(\vartheta_{1}, \ldots, \vartheta_{n}\right)$, then

$$
\mathbf{u}(x+t \vartheta) \cdot \vartheta=\sum_{h=1}^{n} u^{h}(x+t \vartheta) \vartheta_{h} \quad \text { and } \quad \mathcal{E} \mathbf{u}(x+t \vartheta) \vartheta \cdot \vartheta=\sum_{h, k=1}^{n} u_{x_{k}}^{h}(x+t \vartheta) \vartheta_{h} \vartheta_{k}
$$

Indeed, $\sum_{h, k=1}^{n} u_{x_{k}}^{h}(x+t \vartheta) \vartheta_{h} \vartheta_{k}$ agrees with the quadratic form associated with the matrix $\nabla \mathbf{u}(x+t \vartheta)$, evaluated at $\vartheta$. Therefore, it agrees with the quadratic form associated with the symmetric part $\mathcal{E} \mathbf{u}(x+t \vartheta)$ of this matrix, evaluated at $\vartheta$, namely with $\mathcal{E} \mathbf{u}(x+t \vartheta) \vartheta \cdot \vartheta$.

Next, let $\Omega^{\prime}$ be any smooth open set, starshaped with respect to $x$, and such that $\Omega^{\prime} \subset \subset \Omega$. Then the restriction of the function $\mathbf{u}$ to $\Omega^{\prime}$ can be extended to a function in $E^{1,1} \mathbb{R}^{n}$ ), still denoted by $\mathbf{u}$, with compact support - see e.g. [51, Remark 1.3, Chapter 2]. By [51, Proposition 1.3, Chapter 1], there exists a sequence of functions $\left\{\mathbf{u}_{m}\right\} \subset C_{0}^{\infty}\left(\mathbb{R}^{n}\right)$ such that $\mathbf{u}_{m} \rightarrow \mathbf{u}$ in $E^{1,1}\left(\mathbb{R}^{n}\right)$, and $\mathbf{u}_{m} \rightarrow \mathbf{u}$ and $\mathcal{E} \mathbf{u}_{m} \rightarrow \mathcal{E} \mathbf{u}$ a.e. in $\mathbb{R}^{n}$. In particular, $\left\{\mathbf{u}_{m}\right\}$ is a Cauchy sequence in $E^{1,1}\left(\mathbb{R}^{n}\right)$. Given $\varepsilon>0$, one can hence make use of polar coordinates centered at $x$ and of 2.13 to deduce that

$$
\begin{aligned}
\int_{0}^{\infty} t^{n-1} \int_{\mathbb{S}^{n-1}}\left|\mathbf{u}_{m}(x+t \vartheta) \cdot \vartheta-\mathbf{u}_{j}(x+t \vartheta) \cdot \vartheta\right| d \mathcal{H}^{n-1}(\vartheta) d t \\
\quad+\int_{0}^{\infty} t^{n-1} \int_{\mathbb{S}^{n-1}}\left|\frac{d}{d t} \mathbf{u}_{m}(x+t \vartheta) \cdot \vartheta-\frac{d}{d t} \mathbf{u}_{j}(x+t \vartheta) \cdot \vartheta\right| d \mathcal{H}^{n-1}(\vartheta) d t \\
\quad=\int_{0}^{\infty} t^{n-1} \int_{\mathbb{S}^{n-1}}\left|\mathbf{u}_{m}(x+t \vartheta) \cdot \vartheta-\mathbf{u}_{j}(x+t \vartheta) \cdot \vartheta\right| d \mathcal{H}^{n-1}(\vartheta) d t
\end{aligned}
$$




$$
\begin{aligned}
& +\int_{0}^{\infty} t^{n-1} \int_{\mathbb{S}^{n-1}}\left|\sum_{h, k=1}^{n}\left(u_{m}\right)_{x_{k}}^{h}(x+t \vartheta) \vartheta_{h} \vartheta_{k}-\sum_{h, k=1}^{n}\left(u_{j}\right)_{x_{k}}^{h}(x+t \vartheta) \vartheta_{h} \vartheta_{k}\right| d \mathcal{H}^{n-1}(\vartheta) d t \\
= & \int_{0}^{\infty} t^{n-1} \int_{\mathbb{S}^{n-1}}\left|\mathbf{u}_{m}(x+t \vartheta) \cdot \vartheta-\mathbf{u}_{j}(x+t \vartheta) \cdot \vartheta\right| d \mathcal{H}^{n-1}(\vartheta) d t \\
& +\int_{0}^{\infty} t^{n-1} \int_{\mathbb{S}^{n-1}}\left|\left(\mathcal{E} \mathbf{u}_{m}(x+t \vartheta)-\mathcal{E} \mathbf{u}_{j}(x+t \vartheta)\right) \vartheta \cdot \vartheta\right| d \mathcal{H}^{n-1}(\vartheta) d t \\
\leq & \int_{0}^{\infty} t^{n-1} \int_{\mathbb{S}^{n-1}}\left|\mathbf{u}_{m}(x+t \vartheta) \cdot \vartheta-\mathbf{u}_{j}(x+t \vartheta) \cdot \vartheta\right| d \mathcal{H}^{n-1}(\vartheta) d t \\
& +C \int_{0}^{\infty} t^{n-1} \int_{\mathbb{S}^{n-1}}\left|\mathcal{E} \mathbf{u}_{m}(x+t \vartheta)-\mathcal{E} \mathbf{u}_{j}(x+t \vartheta)\right| d \mathcal{H}^{n-1}(\vartheta) d t \\
\leq & C\left\|\mathbf{u}_{m}-\mathbf{u}_{j}\right\|_{E^{1,1}\left(\mathbb{R}^{n}\right)}<\varepsilon,
\end{aligned}
$$

for some constant $C=C(n)$, provided that $m$ and $j$ are large enough. Hence, owing to Fubini's theorem, for $\mathcal{H}^{n-1}$-a.e. $\vartheta \in \mathbb{S}^{n-1}$,

$$
\begin{aligned}
& \int_{0}^{\infty} t^{n-1}\left|\mathbf{u}_{m}(x+t \vartheta) \cdot \vartheta-\mathbf{u}_{j}(x+t \vartheta) \cdot \vartheta\right| d t \\
& \quad+\int_{0}^{\infty} t^{n-1}\left|\frac{d}{d t} \mathbf{u}_{m}(x+t \vartheta) \cdot \vartheta-\frac{d}{d t} \mathbf{u}_{j}(x+t \vartheta) \cdot \vartheta\right| d t<\varepsilon,
\end{aligned}
$$

provided that $m$ and $j$ are large enough. Consequently, for a.e. $\vartheta \in \mathbb{S}^{n-1}$ the sequence $\left\{\mathbf{u}_{m}(x+\right.$ $t \vartheta) \cdot \vartheta\}$ is a Cauchy sequence in $W^{1,1}(\delta, \infty)$ for any $\delta>0$. Hence, there exists a function $\varphi_{x, \theta} \in W_{\text {loc }}^{1,1}(0, \infty)$ such that

$$
\mathbf{u}_{m}(x+t \vartheta) \cdot \vartheta \rightarrow \varphi_{x, \theta}(t)
$$

in $W^{1,1}(\delta, \infty)$. Moreover, on taking a subsequence if necessary, we may assume that equation (2.16) holds for a.e. $t \in(0, \infty)$ and that

$$
\frac{d}{d t} \mathbf{u}_{m}(x+t \vartheta) \cdot \vartheta \rightarrow \frac{d}{d t} \varphi_{x, \theta}(t)
$$

for a.e. $t \in(0, \infty)$. On the other hand, since $\mathbf{u}_{m} \rightarrow \mathbf{u}$ and $\mathcal{E} \mathbf{u}_{m} \rightarrow \mathcal{E} \mathbf{u}$ a.e. in $\mathbb{R}^{n}$, by Fubini's theorem again, for $\mathcal{H}^{n-1}$-a.e. $\vartheta \in \mathbb{S}^{n-1}$,

$$
\mathbf{u}_{m}(x+t \vartheta) \cdot \vartheta \rightarrow \mathbf{u}(x+t \vartheta) \cdot \vartheta
$$

and

$$
\frac{d}{d t} \mathbf{u}_{m}(x+t \vartheta) \cdot \vartheta=\mathcal{E} \mathbf{u}_{m}(x+t \vartheta) \vartheta \cdot \vartheta \rightarrow \mathcal{E} \mathbf{u}(x+t \vartheta) \vartheta \cdot \vartheta
$$

for a.e. $t \in(0, \infty)$. Equations 2.16$)-2.19$ ensure that, for $\mathcal{H}^{n-1}$-a.e. $\vartheta \in \mathbb{S}^{n-1}$,

$$
\varphi_{x, \theta}(t)=\mathbf{u}(x+t \vartheta) \cdot \vartheta \quad \text { and } \quad \frac{d}{d t} \varphi_{x, \theta}(t)=\mathcal{E} \mathbf{u}(x+t \vartheta) \vartheta \cdot \vartheta
$$

for a.e. $t \in(0, \infty)$. Hence, our claim follows, since $\Omega_{x}$ can be invaded by sets $\Omega^{\prime}$ as above.

Now, let $\left\{\vartheta^{1}, \ldots, \vartheta^{n}\right\} \subset \mathbb{S}^{n-1}$ be a basis for $\mathbb{R}^{n}$. For fixed $i \in\{1, \ldots, n\}$, define the function $\varphi^{i}:\left[0, \mathfrak{b}\left(x, \vartheta^{i}\right)\right] \rightarrow \mathbb{R}$ as

$$
\varphi^{i}(t)=\mathbf{u}\left(x+t \vartheta^{i}\right) \cdot \vartheta^{i} \quad \text { for } t \in\left[0, \mathfrak{b}\left(x, \vartheta^{i}\right)\right]
$$


In view of the property established above, for $\mathcal{H}^{n-1}$-a.e. $\vartheta^{1}, \cdots, \vartheta^{n} \in \mathbb{S}^{n-1}$ forming a basis in $\mathbb{R}^{n}$, the function $\varphi^{i}$ is locally absolutely continuous, and

$$
\frac{d \varphi^{i}(t)}{d t}=\mathcal{E} \mathbf{u}\left(x+t \vartheta^{i}\right) \vartheta^{i} \cdot \vartheta^{i} \quad \text { for } t \in\left[0, \mathfrak{b}\left(x, \vartheta^{i}\right)\right] .
$$

Thus, since $\varphi^{i}\left(\mathfrak{b}\left(x, \vartheta^{i}\right)\right)=\mathbf{u}\left(\zeta\left(x, \vartheta^{i}\right)\right) \cdot \vartheta^{i}$,

$$
\mathbf{u}(x) \cdot \vartheta^{i}=\mathbf{u}\left(\zeta\left(x, \vartheta^{i}\right)\right) \cdot \vartheta^{i}-\int_{0}^{\mathfrak{b}\left(x, \vartheta^{i}\right)} \mathcal{E} \mathbf{u}\left(x+t \vartheta^{i}\right) \vartheta^{i} \cdot \vartheta^{i} d t,
$$

whence

$$
\left|\mathbf{u}(x) \cdot \vartheta^{i}\right| \leq\left|\mathbf{u}\left(\zeta\left(x, \vartheta^{i}\right)\right)\right|+C \int_{0}^{\mathfrak{b}\left(x, \vartheta^{i}\right)}\left|\mathcal{E} \mathbf{u}\left(x+t \vartheta^{i}\right)\right| d t
$$

for some constant $C=C(n)$. We now exploit an argument from the proof of [8, Lemma I.2]. On setting

$$
a_{h k}=\sum_{i=1}^{n} \vartheta_{h}^{i} \vartheta_{k}^{i} \quad \text { for } h, k=1, \ldots, n,
$$

one has that

$$
\sum_{i=1}^{n}\left(\mathbf{u}(x) \cdot \vartheta^{i}\right)^{2}=\sum_{h, k=1}^{n} u^{h}(x) u^{k}(x) a_{h k},
$$

a quadratic form in $\mathbf{u}(x)$ associated with the matrix $\mathbf{A}=\left\{a_{h k}\right\}$. Inasmuch as $\left\{\vartheta^{1}, \ldots, \vartheta^{n}\right\}$ is a basis in $\mathbb{R}^{n}$, this quadratic form is positive definite. Furthermore, since the coefficients of its matrix $\mathbf{A}$ depend continuously on the unit vectors $\vartheta^{1}, \cdots, \vartheta^{n}$, its smallest eigenvalue, which agrees with the minimum of the quadratic form on the unit sphere, admits a positive lower bound $\lambda(K)$ as $\left(\vartheta^{1}, \ldots, \vartheta^{n}\right)$ ranges in a compact set $K \subset\left(\mathbb{R}^{n}\right)^{n}$. Therefore,

$$
\lambda(K)^{\frac{1}{2}}|\mathbf{u}(x)| \leq\left(\sum_{i=1}^{n}\left(\mathbf{u}(x) \cdot \vartheta^{i}\right)^{2}\right)^{\frac{1}{2}} \leq \sum_{i=1}^{n}\left|\mathbf{u}(x) \cdot \vartheta^{i}\right| .
$$

As a consequence, inequality (2.21) implies that

$$
\lambda(K)^{\frac{1}{2}}|\mathbf{u}(x)| \leq \sum_{i=1}^{n}\left|\mathbf{u}\left(\zeta\left(x, \vartheta^{i}\right)\right)\right|+\sum_{i=1}^{n} \int_{0}^{\mathfrak{b}\left(x, \vartheta^{i}\right)}\left|\mathcal{E} \mathbf{u}\left(x+t \vartheta^{i}\right)\right| d t .
$$

Next, set $\eta=\left(\eta_{1}, \ldots \eta_{n-1}\right)$,

$$
Q=\left\{\eta \in \mathbb{R}^{n-1}: 0 \leq \eta_{i} \leq 1, i=1, \ldots, n-1\right\},
$$

and consider the functions $\phi_{i}: Q \rightarrow \mathbb{R}^{n}$, with $i=1, \ldots, n$, defined as

$$
\phi_{i}(\eta)= \begin{cases}\left(\eta_{1}, \ldots, \eta_{i-1}, \eta_{i}+1, \eta_{i+1}, \ldots, \eta_{n-1}, 0\right) & \text { if } i=1, \ldots, n-1, \\ \left(\eta_{1}, \ldots, \eta_{n-1}, 0\right) & \text { if } i=n,\end{cases}
$$

for $\eta \in Q$. Set $x_{0}=(1,1, \ldots, 1) \in \mathbb{R}^{n}$, and define the functions $\Phi_{i}: Q \rightarrow \mathbb{S}^{n-1}, i=1, \ldots, n$, as

$$
\Phi_{i}(\eta)=\frac{\phi_{i}(\eta)-x_{0}}{\left|\phi_{i}(\eta)-x_{0}\right|} \quad \text { for } \eta \in Q
$$


For each index $i$, the function $\Phi_{i}: Q \rightarrow \Phi_{i}(Q)$ is a local coordinate system on $\mathbb{S}^{n-1}$. Moreover, the set $\left\{\Phi_{1}(\eta), \ldots, \Phi_{n}(\eta)\right\}$ is a basis of unit vectors in $\mathbb{R}^{n}$ for each $\eta \in Q$, and the image of the map

$$
Q \ni \eta \mapsto\left(\Phi_{1}(\eta), \ldots, \Phi_{n}(\eta)\right) \in\left(\mathbb{R}^{n}\right)^{n}
$$

is a compact subset of $\left(\mathbb{R}^{n}\right)^{n}$. Thereby, an application of inequality 2.22 with $\vartheta^{i}=\Phi_{i}(\eta)$ implies that

$$
c|\mathbf{u}(x)| \leq \sum_{i=1}^{n}\left|\mathbf{u}\left(\zeta\left(x, \Phi_{i}(\eta)\right)\right)\right|+\sum_{i=1}^{n} \int_{0}^{\mathfrak{b}\left(x, \Phi_{i}(\eta)\right)}\left|\mathcal{E} \mathbf{u}\left(x+t \Phi_{i}(\eta)\right)\right| d t \quad \text { for } \eta \in Q,
$$

for a suitable constant $c=c(n)$. A change of variables ensures that

$$
\int_{Q}\left|\mathbf{u}\left(\zeta\left(x, \Phi_{i}(\eta)\right)\right)\right| d \eta \leq C \int_{\mathbb{S}^{n-1}}|\mathbf{u}(\zeta(x, \vartheta))| d \mathcal{H}^{n-1}(\vartheta)
$$

for some constant $C=C(n)$, and for $i=1, \ldots, n$. Analogously,

$$
\begin{aligned}
\int_{Q} \int_{0}^{\mathfrak{b}\left(x, \Phi_{i}(\eta)\right)} \mid & \mathcal{E} \mathbf{u}\left(x+t \Phi_{i}(\eta)\right)\left|d t d \eta \leq C \int_{\mathbb{S}^{n-1}} \int_{0}^{\mathfrak{b}(x, \vartheta)}\right| \mathcal{E} \mathbf{u}(x+t \vartheta) \mid d t d \mathcal{H}^{n-1}(\vartheta) \\
& =C \int_{\mathbb{S}^{n-1}} \int_{0}^{\mathfrak{b}(x, \vartheta)} t^{n-1} \frac{|\mathcal{E} \mathbf{u}(x+t \vartheta)|}{t^{n-1}} d t d \mathcal{H}^{n-1}(\vartheta) \leq C^{\prime} \int_{\Omega_{x}} \frac{|\mathcal{E} \mathbf{u}(y)|}{|x-y|^{n-1}} d y
\end{aligned}
$$

for some constants $C=C(n), C^{\prime}=C^{\prime}(n)$ and for $i=1, \ldots, n$. Inequality 2.10 follows on integrating inequality 2.23 ) over $Q$, and exploiting equations (2.24) and (2.25).

\section{A rearrangement inequality and an ensuing reduction princi- ple}

The pointwise bound established in the previous section enables us to derive an estimate, in rearrangement form, with respect to any $\alpha$-upper Ahlfors regular measure - also called Frostman measure $-\mu$ on $\Omega$, with $\alpha \in(n-1, n]$. Namely, a Borel measure $\mu$ such that

$$
\mu\left(B_{r}(x) \cap \Omega\right) \leq C_{\mu} r^{\alpha} \quad \text { for } x \in \Omega \text { and } r>0,
$$

for some exponent $\alpha \in(n-1, n]$ and some constant $C_{\mu}>0$. Here, $B_{r}(x)$ denotes the ball, centered at $x$, with radius $r$.

Given a measure space $\mathcal{R}$, endowed with a $\sigma$-finite, non-atomic measure $\nu$, the decreasing rearrangement $\phi_{\nu}^{*}:[0, \infty) \rightarrow[0, \infty]$ of a $\nu$-measurable function $\phi: \mathcal{R} \rightarrow \mathbb{R}$ is defined as

$$
\phi_{\nu}^{*}(s)=\inf \{t \geq 0: \nu(\{|\phi|>t\}) \leq s\} \quad \text { for } s \in[0, \infty) .
$$

Although nonlinear, the operation of decreasing rearrangement has the property that

$$
(\phi+\psi)_{\nu}^{*}(s) \leq \phi_{\nu}^{*}(s / 2)+\psi_{\nu}^{*}(s / 2) \quad \text { for } s \geq 0,
$$

for every measurable functions $\phi$ and $\psi$ on $\mathcal{R}$.

Integrability properties of a function $\phi$ are preserved under the operation of decreasing rearrangement, since the functions $\phi$ and $\phi_{\nu}^{*}$ share the same distribution function. Indeed,

$$
\nu(\{|\phi|>t\})=\mathcal{L}^{1}\left(\left\{\phi_{\nu}^{*}>t\right\}\right) \text { for every } t \geq 0 .
$$


Theorem 3.1 [Rearrangement estimate] Let $\Omega$ be any open set in $\mathbb{R}^{n}, n \geq 2$. Assume that $\mu$ is a Borel measure in $\Omega$ fulfilling (3.1) for some exponent $\alpha \in(n-1, n]$ and for some $C_{\mu}>0$. Then there exists constants $c=c(n)$ and $C=C\left(n, \alpha, C_{\mu}\right)$ such that

$$
\begin{array}{r}
|\mathbf{u}|_{\mu}^{*}(c s) \leq C\left[s^{-\frac{n-1}{\alpha}} \int_{0}^{s^{\frac{n}{\alpha}}}|\mathcal{E} \mathbf{u}|_{\mathcal{L}^{n}}^{*}(r) d r+\int_{s^{\frac{n}{\alpha}}}^{\infty} r^{-\frac{n-1}{n}}|\mathcal{E} \mathbf{u}|_{\mathcal{L}^{n}}^{*}(r) d r\right. \\
\left.+s^{-\frac{n-1}{\alpha}} \int_{0}^{s^{\frac{n-1}{\alpha}}}\left|\mathbf{u}_{\mid \partial \Omega}\right|_{\mathcal{H}^{n-1}}^{*}(r) d r\right] \quad \text { for } s>0,
\end{array}
$$

for every function $\mathbf{u} \in E^{1,1}(\Omega) \cap C_{b}(\bar{\Omega})$.

Proof. Let us denote by $I$ the classical Riesz potential operator of order 1, given by

$$
I f(x)=\int_{\Omega} \frac{f(y)}{|y-x|^{n-1}} d y \quad \text { for } x \in \Omega,
$$

at any $f \in L^{1}(\Omega)$. By [16, Lemma 7.6], there exists a constant $C=C\left(n, \alpha, C_{\mu}\right)$, such that

$$
(I f)_{\mu}^{*}(s) \leq C\left(s^{-\frac{n-1}{\alpha}} \int_{0}^{s^{\frac{n}{\alpha}}} f_{\mathcal{L}^{n}}^{*}(r) d r+\int_{s^{\frac{n}{\alpha}}}^{\infty} r^{-\frac{n-1}{n}} f_{\mathcal{L}^{n}}^{*}(r) d r\right) \quad \text { for } s>0,
$$

for every $f \in L^{1}(\Omega)$. Next, define the operator $T$ as

$$
T g(x)=\int_{\mathbb{S}^{n-1}}|g(\zeta(x, \vartheta))| d \mathcal{H}^{n-1}(\vartheta) \quad \text { for } x \in \Omega
$$

at any function Borel function $g: \partial \Omega \rightarrow \mathbb{R}$. Here, and in what follows, we adopt convention (2.7). Note that, owing to Fubini's theorem, $T g$ is a measurable function with respect to any Borel measure in $\Omega$. By [16, Lemma 7.5], there exists a constant $C=C\left(n, \alpha, C_{\mu}\right)$ such that

$$
(T g)_{\mu}^{*}(s) \leq C s^{-\frac{n-1}{\alpha}} \int_{0}^{s^{\frac{n-1}{\alpha}}} g_{\mathcal{H}^{n-1}}^{*}(r) d r \quad \text { for } s>0,
$$

for every Borel function $g: \partial \Omega \rightarrow \mathbb{R}$.

With notations (3.4) and (3.6) in force, inequality (2.10) takes the form

$$
|\mathbf{u}(x)| \leq C(T|\mathbf{u}|(x)+I|\mathcal{E} \mathbf{u}|(x)) \quad \text { for } x \in \Omega .
$$

Hence, inequality (3.3) follows via inequalities $(3.5)$ and $(3.7)$, thanks to property $(3.2)$ of rearrangements.

Theorem 3.1 is the key step in a proof of the reduction principle contained in Theorem 3.2 below. The latter enables one to derive norm inequalities of the form (1.4) from corresponding one-dimensional inequalities for Hardy type operators dictated by the right-hand side of inequality (3.3). Norms in rearrangement-invariant spaces are allowed in Theorem 3.2. Recalll that a rearrangement-invariant space $X(\mathcal{R})$, on a measure space $\mathcal{R}$ as above, is a Banach function space (in the sense of Luxemburg) endowed with a norm $\|\cdot\|_{X(\mathcal{R})}$ such that

$$
\|\phi\|_{X(\mathcal{R})}=\|\psi\|_{X(\mathcal{R})} \quad \text { whenever } \quad \phi_{\nu}^{*}=\psi_{\nu}^{*} .
$$


Every rearrangement-invariant space $X(\mathcal{R})$ admits a representation space $\bar{X}(0, \infty)$, namely another rearrangement-invariant space on $(0, \infty)$ such that

$$
\|\phi\|_{X(\mathcal{R})}=\left\|\phi_{\nu}^{*}\right\|_{\bar{X}(0, \infty)} \quad \text { for every } \phi \in X(\mathcal{R}) .
$$

In customary situations, an expression for the norm $\|\cdot\|_{\bar{X}(0, \infty)}$ immediately follows from that of $\|\cdot\|_{X(\mathcal{R})}$. Lebesgue, Lorentz and Orlicz spaces are classical instances of rearrangement-invariant spaces. We refer to [3] for a comprehensive account of rearrangement-invariant spaces.

Theorem 3.2 [Reduction principle] Let $\Omega$ be any open set in $\mathbb{R}^{n}, n \geq 2$. Assume that $\mu$ is a Borel measure in $\Omega$ fulfilling (3.1) for some exponent $\alpha \in(n-1, n]$ and for some constant $C_{\mu}$. Let $X(\Omega), Y(\Omega, \mu)$ and $Z(\partial \Omega)$ be rearrangement-invariant spaces such that

$$
\begin{gathered}
\left\|s^{-\frac{n-1}{\alpha}} \int_{0}^{s^{\frac{n}{\alpha}}} \varphi(r) d r\right\|_{\bar{Y}(0, \infty)} \leq C\|\varphi\|_{\bar{X}(0, \infty)}, \\
\left\|\int_{s^{\frac{n}{\alpha}}}^{\infty} r^{-\frac{n-1}{n}} \varphi(r) d r\right\|_{\bar{Y}(0, \infty)} \leq C\|\varphi\|_{\bar{X}(0, \infty)}, \\
\left\|s^{-\frac{n-1}{\alpha} \int_{0}^{s^{\frac{n-1}{\alpha}}} \varphi(r) d r}\right\|_{\bar{Y}(0, \infty)} \leq C\|\varphi\|_{\bar{Z}(0, \infty)},
\end{gathered}
$$

for some constant $C$, and for every non-increasing function $\varphi:[0, \infty) \rightarrow[0, \infty)$. Then there exists a constant $C^{\prime}=C^{\prime}\left(n, \alpha, C_{\mu}, C\right)$ such that

$$
\|\mathbf{u}\|_{Y(\Omega, \mu)} \leq C^{\prime}\left(\|\mathcal{E} \mathbf{u}\|_{X(\Omega)}+\|\mathbf{u}\|_{Z(\partial \Omega)}\right)
$$

for every function $u \in E^{1} X(\Omega) \cap C_{b}(\bar{\Omega})$.

Remark 3.3 Assumptions (3.11)- 3.13) of Theorem 3.2 can be weakened if either $\mu(\Omega)<\infty$, or $\mathcal{L}^{n}(\Omega)<\infty$, or $\mathcal{H}^{n-1}(\partial \Omega)<\infty$. Specifically: if $\mu(\Omega)<\infty$, it suffices to assume that there exists $L \in(0, \infty)$ such that inequalities (3.11) $-(3.13)$ hold with the integral operators multiplied by $\chi_{(0, L)}$ on the left-hand sides; if $\mathcal{L}^{n}(\Omega)<\infty$, it suffices to assume that inequalities (3.11) (3.12 hold with $\varphi$ replaced by $\varphi \chi_{(0, M)}$ for some $M \in(0, \infty)$; if $\mathcal{H}^{n-1}(\partial \Omega)<\infty$, it suffices to assume that inequality (3.13) holds with $\varphi$ replaced by $\varphi \chi_{(0, N)}$ for some $N \in(0, \infty)$. After these modifications in the assumptions, inequality (3.14) still holds, but with $C^{\prime}$ depending also on either on $L$ and $\mu(\Omega)$, or on $M$ and $\mathcal{L}^{n}(\Omega)$, or on $N$ and $\mathcal{H}^{n-1}(\partial \Omega)<\infty$, according to whether $\mu(\Omega)<\infty$, or $\mathcal{L}^{n}(\Omega)<\infty$, or $\mathcal{H}^{n-1}(\partial \Omega)<\infty$.

Proof of Theorem 3.2. Let $u$ be any function as in the statement. Inequalities (3.3) and (3.11) - 3.13) imply that

$$
\left\||\mathbf{u}|_{\mu}^{*}(c s)\right\|_{\bar{Y}(0, \infty)} \leq C\left[\left\|s^{-\frac{n-1}{\alpha}} \int_{0}^{s^{\frac{n}{\alpha}}}\left|\mathcal{E} \mathbf{u}_{\mathcal{L}^{n}}^{*}(r) d r\left\|_{\bar{Y}(0, \infty)}+\right\| \int_{s^{\frac{n}{\alpha}}}^{\infty} r^{-\frac{n-1}{n}}\right| \mathcal{E} \mathbf{u}_{\mathcal{L}^{n}}^{*}(r) d r\right\|_{\bar{Y}(0, \infty)}\right.
$$




$$
\begin{aligned}
& \left.+\left\|s^{-\frac{n-1}{\alpha}} \int_{0}^{s^{\frac{n-1}{\alpha}}}\left|\mathbf{u}_{\mid \partial \Omega}\right|_{\mathcal{H}^{n-1}}^{*}(r) d r\right\|_{\bar{Y}(0, \infty)}\right] \\
\leq & C^{\prime}\left[\left\||\mathcal{E} \mathbf{u}|_{\mathcal{L}^{n}}^{*}(s)\right\|_{\bar{X}(0, \infty)}+\left\|\left|\mathbf{u}_{\mid \partial \Omega}\right|_{\mathcal{H}^{n-1}}^{*}(s)\right\|_{\bar{Z}(0, \infty)}\right],
\end{aligned}
$$

where $c=c(n), C=C\left(n, \alpha, C_{\mu}\right)$ and $C^{\prime}$ depends on $n, \alpha, C_{\mu}$ and on the constant $C$ appearing in (3.11)-(3.13). On the other hand, a property of rearrangement-invariant norms under dilations [3. Proposition 5.11, Chapter 3] tells us that the norm on the left-hand side of inequality (3.15) is bounded from below by $\min \left\{1, \frac{1}{c}\right\}|||\mathbf{u}|_{\mu}^{*}(s) \|_{\bar{Y}(0, \infty)}$. Thereby, one infers from 3.15 and 3.10) that

$$
\|\mathbf{u}\|_{Y(\Omega, \mu)} \leq C\left(\|\mathcal{E} \mathbf{u}\|_{X(\Omega)}+\|\mathbf{u}\|_{Z(\partial \Omega)}\right)
$$

for some constant $C$ depending on $n, \alpha, C_{\mu}$ and on the constant $C$ in (3.11)-(3.13). Inequality (3.14) follows.

\section{Sobolev type inequalities}

In this section we exhibit a few e inequalities of the form (1.4), which can be established via Theorem 3.2. Inequalities involving Lebesgue norms, Lorentz norms, and Orlicz norms of exponential or power-logarithmic type, which naturally come into play in borderline situations, will be presented. All inequalities are stated and proved for general $\alpha$-upper Ahlfors regular measures $\mu$, with $\alpha \in(n-1, n]$. The statements for the standard case of the Lebesgue measure can simply be obtained on setting $\alpha=n$.

The target norm $\|\cdot\|_{Y(\Omega, \mu)}$ appearing in inequality (1.4) depends on both the norm $\| \cdot$ $\|_{X(\Omega)}$ and the norm $\|\cdot\|_{Z(\partial \Omega)}$. In the following discussion, given $\|\cdot\|_{X(\Omega)}$, we limit ourselves to considering the best possible norm $\|\cdot\|_{Z(\partial \Omega)}$, within a prescribed family of norms, in a boundary trace embedding for the space $E^{1} X(\Omega)$. The corresponding strongest possible norm $\|\cdot\|_{Y(\Omega, \mu)}$ in inequality (1.4) is then exhibited. Of course, different choices of the norm $\|\cdot\|_{Z(\partial \Omega)}$ are possible for a given $\|\cdot\|_{X(\Omega)}$. The optimal target norm $\|\cdot\|_{Y(\Omega, \mu)}$ then depends on a balance between $\|\cdot\|_{X(\Omega)}$ and $\|\cdot\|_{Z(\partial \Omega)}$.

We premise some notations and definitions in connection with the norms appearing in our results. Let $\mathcal{R}$ be a measure space equipped with a $\sigma$-finite, non-atomic measure $\nu$. The Orlicz space $L^{A}(\mathcal{R})$ built upon a Young function $A:[0, \infty) \rightarrow[0, \infty]$, namely a left-continuous convex function which is neither identically equal to 0 nor to $\infty$, is a rearrangement-invariant space equipped the Luxemburg norm given by

$$
\|\phi\|_{L^{A}(\mathcal{R})}=\inf \left\{\lambda>0: \int_{\mathcal{R}} A\left(\frac{|\phi(x)|}{\lambda}\right) d x \leq 1\right\}
$$

for a measurable function $\phi$ in $\mathcal{R}$. The class of Orlicz spaces includes that of Lebesgue spaces, since $L^{A}(\mathcal{R})=L^{p}(\mathcal{R})$ if $A(t)=t^{p}$ for $p \in[1, \infty)$, and $L^{A}(\mathcal{R})=L^{\infty}(\mathcal{R})$ if $A(t)=0$ for $t \in[0,1]$ and $A(t)=\infty$ for $t \in(1, \infty)$. Given $\sigma>0$, we denote by $\exp L^{\sigma}(\mathcal{R})$ and $\exp \exp L^{\sigma}(\mathcal{R})$ the Orlicz spaces built upon Young functions equivalent to the functions $A(t)=e^{t^{\sigma}}-1$ and $A(t)=e^{e^{t^{\sigma}}}-e$, respectively, near inifnity. If $p>1$ and $\sigma \in \mathbb{R}$, the notation $L^{p}(\log L)^{\sigma}(\mathcal{R})$ stands for the Orlicz space, also called Zygmund space, built upon a Young function equivalent to the function $A(t)=t^{p} \log ^{\sigma}(1+t)$ near infinity. 
The Lorentz norms depend on two parameters, say $p$ and $q$. Assume that either $1<p<\infty$ and $1 \leq q \leq \infty$, or $p=q=1$, or $p=q=\infty$. We define the functional $\|\cdot\|_{L^{p, q}(\mathcal{R})}$ by

$$
\|\phi\|_{L^{p, q}(\mathcal{R})}=\left\|s^{\frac{1}{p}-\frac{1}{q}} \phi_{\nu}^{*}(s)\right\|_{L^{q}(0, \infty)}
$$

for a measurable function $\phi$ in $\mathcal{R}$. Then $\|\cdot\|_{L^{p, q}(\mathcal{R})}$ is equivalent to a rearrangement-invariant norm. The corresponding space $L^{p, q}(R)$ is called Lorentz space.

Suppose now that $1<q<\infty$ and $\nu(\mathcal{R})<\infty$. The Lorentz-Zygmund space $L^{\infty, q ;-1}(\mathcal{R})$ is defined via the rearrangement-invariant norm given by

$$
\|\phi\|_{L^{\infty, q ;-1}(\mathcal{R})}=\| s^{-\frac{1}{q} \log ^{-1}\left(1+\frac{\nu(\mathcal{R})}{s}\right) \phi_{\nu}^{*}(s) \|_{L^{q}(0, \nu(\mathcal{R}))}}
$$

for a measurable function $\phi$ in $\mathcal{R}$.

Theorem 4.1 [Subcritical Sobolev inequality] Let $\Omega$ be any open set in $\mathbb{R}^{n}, n \geq 2$. Assume that $\mu$ is a measure in $\Omega$ fulfilling (3.1) for some exponent $\alpha \in(n-1, n]$ and for some constant $C_{\mu}$. If $1<p<n$, then there exists a constant $C=C\left(n, p, \alpha, C_{\mu}\right)$ such that

$$
\|\mathbf{u}\|_{L^{\frac{\alpha p}{n-p}}(\Omega, \mu)} \leq C\left(\|\mathcal{E} \mathbf{u}\|_{L^{p}(\Omega)}+\|\mathbf{u}\|_{L^{\frac{p(n-1)}{n-p}}(\partial \Omega)}\right)
$$

for every $u \in E^{1, p}(\Omega) \cap C_{b}(\bar{\Omega})$.

Proof. If $\mathcal{R}$ is any $\sigma$-finite, non-atomic measure space, the space $L^{p}(0, \infty)$ is a representation space of the Lebesgue space $L^{p}(\mathcal{R})$. Inequality (4.3) then follows from Theorem 3.2 , via standard one-dimensional Hardy type inequalities for Lebesgue norms (see e.g. [43, Section 1.3.2]).

The next result tells us that, as in the classical Rellich theorem, the Sobolev embedding corresponding to inequality 4.3 is pre-compact if the exponent $\frac{\alpha p}{n-p}$ is replaced with any smaller one, and $\mu(\Omega)<\infty$.

Theorem 4.2 [Pre-compact Sobolev embedding] Let $\Omega, \mu$ and $p$ be as in Theorem 4.1. Assume, in addition, that $\mu(\Omega)<\infty$. If $1 \leq q<\frac{\alpha p}{n-p}$, and $\left\{\mathbf{u}_{m}\right\}$ is a bounded sequence in $E^{1, p}(\Omega) \cap L^{\frac{p(n-1)}{n-p}}(\partial \Omega)$, then $\left\{\mathbf{u}_{m}\right\}$ is a Cauchy sequence in $L^{q}(\Omega, \mu)$.

Proof. Fix any $\varepsilon>0$, and choose a compact set $K \subset \Omega$ such that $\mu(\Omega \backslash K)<\varepsilon$. Let $\varrho \in C_{0}^{\infty}(\Omega)$ be such that $0 \leq \varrho \leq 1$ and $\varrho=1$ in $K$. Thus, $K \subset \operatorname{supp}(\varrho)$, the support of $\varrho$, and hence

$$
\mu(\operatorname{supp}(1-\varrho)) \leq \mu(\Omega \backslash K)<\varepsilon
$$

Let $\Omega^{\prime}$ be an open set, with a smooth boundary, satisfying $\operatorname{supp}(\varrho) \subset \Omega^{\prime} \subset \Omega$. Let $\left\{\mathbf{u}_{m}\right\}$ be a bounded sequence in $E^{1, p}(\Omega) \cap L^{\frac{p(n-1)}{n-p}}(\partial \Omega)$. An application of Theorem 4 .1 with $\mu=\mathcal{L}^{n}$, tells us that $\left\{\mathbf{u}_{m}\right\}$ is also bounded in $L^{p}\left(\Omega^{\prime}\right)$. As recalled in Section 1 , thanks to a version of the Korn inequality, the space $E^{1, p}\left(\Omega^{\prime}\right) \cap L^{p}\left(\Omega^{\prime}\right)$ agrees with the standard Sobolev space $W^{1, p}\left(\Omega^{\prime}\right)$, up to equivalent norms. By a weighted version of Rellich's compactness theorem [43, Theorem 1.4.6/1], $\left\{\mathbf{u}_{m}\right\}$ is a Cauchy sequence in $L^{q}\left(\Omega^{\prime}, \mu\right)$, and hence there exists $m_{0} \in \mathbb{N}$ such that

$$
\left\|\mathbf{u}_{m}-\mathbf{u}_{j}\right\|_{L^{q}\left(\Omega^{\prime}, \mu\right)}<\varepsilon
$$


if $m, j>m_{0}$. By Hölder's inequality,

$$
\begin{aligned}
\left\|(1-\varrho)\left(\mathbf{u}_{m}-\mathbf{u}_{j}\right)\right\|_{L^{q}(\Omega, \mu)} & \leq\left\|\mathbf{u}_{m}-\mathbf{u}_{j}\right\|_{L^{\frac{\alpha p}{n-p}}(\Omega, \mu)} \mu(\operatorname{supp}(1-\varrho))^{\frac{\alpha p-(n-p) q}{\alpha p q}} \\
& \leq C\left(\left\|\mathbf{u}_{m}\right\|_{E^{1, p}(\Omega) \cap L^{\frac{p(n-1)}{n-p}}(\partial \Omega)}+\left\|\mathbf{u}_{j}\right\|_{E^{1, p}(\Omega) \cap L^{\frac{p(n-1)}{n-p}}(\partial \Omega)}\right) \varepsilon^{\frac{\alpha p-(n-p) q}{\alpha p q}} \\
& \leq C^{\prime} \varepsilon^{\frac{\alpha p-(n-p) q}{\alpha p q}}
\end{aligned}
$$

for some constants $C$ and $C^{\prime}$ independent of $m$ and $j$. Inequalities (4.5) and (4.6) tell us that

$$
\left\|\mathbf{u}_{m}-\mathbf{u}_{j}\right\|_{L^{q}(\Omega, \mu)} \leq\left\|\mathbf{u}_{m}-\mathbf{u}_{j}\right\|_{L^{q}\left(\Omega^{\prime}, \mu\right)}+\left\|(1-\varrho)\left(\mathbf{u}_{m}-\mathbf{u}_{j}\right)\right\|_{L^{q}(\Omega, \mu)} \leq \varepsilon+C^{\prime} \varepsilon^{\frac{\alpha p-(n-p) q}{\alpha p q}}
$$

if $m, j>m_{0}$. Owing to the arbitrariness of $\varepsilon$, inequality (4.7) implies that $\left\{\mathbf{u}_{m}\right\}$ is a Cauchy sequence in $L^{q}(\Omega, \mu)$.

The next statement concernes the borderline exponent $p=n$, which is not included in Theorem 4.1. It consists of two inequalities. The former is a version, for the symmetric gradient, of the Yudovich-Pohozaev-Trudinger inequality, in arbitrary domains with an upper Ahlfors regular measure, and involves an Orlicz norm of exponential type. The latter amonts to a slight improvement of the former, in that it allows for a stronger norm in a Lorentz-Zygmund space. It can be regarded as a counterpart in the present framework of a result independently obtained by Hansson and Brezis-Weinger in the classical setting. Interestingly, the norms coming into play in this limiting situation are independent of the exponent $\alpha$ in (3.1), and hence they are the same as for the Lebesgue measure. They also agree with those appearing in the parallel inequalities for the full gradient. By contrast, the exponent $\alpha$ affects the constants in the inequalities in question. Let us incidentally mention that the sharp constant in the exponential YudovichPohozaev-Trudinger inequality, for the standard gradient, is detected in the paper [13], which extends to Ahlfors regular measures a classical inequality by Moser.

Theorem 4.3 [Critical Sobolev inequality] Let $\Omega$ and $\mu$ be as in Theorem 4.1. Assume, in addition, that $\mathcal{L}^{n}(\Omega)<\infty, \mu(\Omega)<\infty$ and $\mathcal{H}^{n-1}(\partial \Omega)<\infty$. Then there exists a constant $C=C\left(n, \alpha, C_{\mu}, \mathcal{L}^{n}(\Omega), \mu(\Omega), \mathcal{H}^{n-1}(\partial \Omega)\right)$ such that

$$
\|\mathbf{u}\|_{\exp L^{\frac{n}{n-1}}(\Omega, \mu)} \leq C\left(\|\mathcal{E} \mathbf{u}\|_{L^{n}(\Omega)}+\|\mathbf{u}\|_{\exp L^{\frac{n}{n-1}(\partial \Omega)}}\right)
$$

for every $u \in E^{1, n}(\Omega) \cap C_{b}(\bar{\Omega})$.

Moreover, there exists a constant as above such that

$$
\|\mathbf{u}\|_{L^{\infty ; n,-1}(\Omega, \mu)} \leq C\left(\|\mathcal{E} \mathbf{u}\|_{L^{n}(\Omega)}+\|\mathbf{u}\|_{L^{\infty ; n,-1}(\partial \Omega)}\right)
$$

for every $u \in E^{1, n}(\Omega) \cap C_{b}(\bar{\Omega})$.

Proof. If $\mathcal{R}$ is a finite measure space, then the norm of a function $\phi$ in the Orlicz space $\exp L^{\sigma}(\mathcal{R})$, with $\sigma>0$, is equivalent, up to multiplicative constants depending on $\sigma$ and $\nu(\mathcal{R})$, to the functional

$$
\left\|\left(1+\log \frac{\nu(\mathcal{R})}{s}\right)^{-\frac{1}{\sigma}} \phi_{\nu}^{*}(s)\right\|_{L^{\infty}(0, \nu(\mathcal{R}))},
$$


see e.g. [3, Lemma 6.12, Chapter 4]. Now, one can verify that the function

$$
(0, \infty) \ni s \mapsto s^{-\frac{n-1}{\alpha}} \int_{0}^{s^{\frac{n}{\alpha}}} \varphi(r) d r+\int_{s^{\frac{n}{\alpha}}}^{\infty} r^{-\frac{n-1}{n}} \varphi(r) d r
$$

is non-decreasing. Also, if $\varphi$ is non-increasing, then the function

$$
(0, \infty) \ni s \mapsto s^{-\frac{n-1}{\alpha}} \int_{0}^{s^{\frac{n-1}{\alpha}}} \varphi(r) d r
$$

is non-increasing as well. By Theorem 3.2 and Remark 3.3 , the proof of inequality $(4.8)$ is thus reduced to showing that

$$
\begin{aligned}
& \left\|s^{-\frac{n-1}{\alpha}}\left(1+\log \frac{\mu(\Omega)}{s}\right)^{-\frac{n-1}{n}} \int_{0}^{s^{\frac{n}{\alpha}}} \varphi(r) d r\right\|_{L^{\infty}(0, \mu(\Omega))} \leq C\|\varphi\|_{L^{n}\left(0, \mathcal{L}^{n}(\Omega)\right)}, \\
& \left\|\left(1+\log \frac{\mu(\Omega)}{s}\right)^{-\frac{n-1}{n}} \int_{s^{\frac{n}{\alpha}}}^{\infty} r^{-\frac{n-1}{n}} \varphi(r) d r\right\|_{L^{\infty}(0, \mu(\Omega))} \leq C\|\varphi\|_{L^{n}\left(0, \mathcal{L}^{n}(\Omega)\right)},
\end{aligned}
$$

for every non-increasing function $\varphi:(0, \infty) \rightarrow[0, \infty)$ with support in $\left(0, \mathcal{L}^{n}(\Omega)\right)$, and

$$
\begin{aligned}
\| s^{-\frac{n-1}{\alpha}}\left(1+\log \frac{\mu(\Omega)}{s}\right)^{-\frac{n-1}{n}} \int_{0}^{s^{\frac{n-1}{\alpha}}} \varphi(r) d r & \|_{L^{\infty}(0, \mu(\Omega))} \\
\leq C & \left(1+\log \frac{\mathcal{H}^{n-1}(\partial \Omega)}{s}\right)^{-\frac{n-1}{n}} \varphi(s) \|_{L^{\infty}\left(0, \mathcal{H}^{n-1}(\partial \Omega)\right)},
\end{aligned}
$$

for some constant $C$ and every non-increasing function for every non-increasing function $\varphi$ : $(0, \infty) \rightarrow[0, \infty)$ with support in $\left(0, \mathcal{H}^{n-1}(\partial \Omega)\right)$

Inequalities 4.12 - 4.14) can be verified via classical characterizations of Hardy type inequalities in weighted Lebesgue spaces [43, Section 1.3.2].

Let us now consider inequality (4.9). By Theorem 3.2 and Remark 3.3 again, it suffices to show that

$$
\left\|s^{-\frac{n-1}{\alpha}} \int_{0}^{s^{\frac{n}{\alpha}}} \varphi(r) d r+\int_{s^{\frac{n}{\alpha}}}^{\infty} r^{-\frac{n-1}{n}} \varphi(r) d r\right\|_{L^{\infty ; n,-1}(0, \mu(\Omega))} \leq C\|\varphi\|_{L^{n}\left(0, \mathcal{L}^{n}(\Omega)\right)},
$$

for some constant $C$ and for every non-increasing function $\varphi:(0, \infty) \rightarrow[0, \infty)$ with support in $\left(0, \mathcal{L}^{n}(\Omega)\right)$, and

$$
\left\|s^{-\frac{n-1}{\alpha}} \int_{0}^{s^{\frac{n-1}{\alpha}}} \varphi(r) d r\right\|_{L^{\infty ; n,-1}(0, \mu(\Omega))} \leq C\|\varphi\|_{L^{\infty ; n,-1}\left(0, \mathcal{H}^{n-1}(\partial \Omega)\right)},
$$

for some constant $C$ and every non-increasing function for every non-increasing function $\varphi$ : $(0, \infty) \rightarrow[0, \infty)$ with support in $\left(0, \mathcal{H}^{n-1}(\partial \Omega)\right)$. As a consequence of the monotonicy of the functions in 4.10 and (4.11), inequality 4.15 is equivalent to the couple of inequalities

$$
\left\|s^{-\frac{n-1}{\alpha}-\frac{1}{n}}\left(1+\log \frac{\mu(\Omega)}{s}\right)^{-1} \int_{0}^{s^{\frac{n}{\alpha}}} \varphi(r) d r\right\|_{L^{n}(0, \mu(\Omega))} \leq C\|\varphi\|_{L^{n}\left(0, \mathcal{L}^{n}(\Omega)\right)},
$$


and

$$
\left\|\int_{s^{\frac{n}{\alpha}}}^{\infty} r^{-\frac{n-1}{n}} \varphi(r) d r\right\|_{L^{\infty ; n,-1}(0, \mu(\Omega))} \leq C\|\varphi\|_{L^{n}\left(0, \mathcal{L}^{n}(\Omega)\right)}
$$

for some constant $C$ and for every non-increasing function $\varphi:(0, \infty) \rightarrow[0, \infty)$ with support in $\left(0, \mathcal{L}^{n}(\Omega)\right)$, and inequality 4.16 is equivalent to the inequality

$$
\left\|s^{-\frac{n-1}{\alpha}-\frac{1}{n}}\left(1+\log \frac{\mu(\Omega)}{s}\right)^{-1} \int_{0}^{s^{\frac{n-1}{\alpha}}} \varphi(r) d r\right\|_{L^{n}(0, \mu(\Omega))} \leq C\|\varphi\|_{L^{\infty ; n,-1}\left(0, \mathcal{H}^{n-1}(\partial \Omega)\right)},
$$

for some constant $C$ and every non-increasing function for every non-increasing function $\varphi$ : $(0, \infty) \rightarrow[0, \infty)$ with support in $\left(0, \mathcal{H}^{n-1}(\partial \Omega)\right)$. Inequalities 4.17$)$ and 4.19 can be derived as special cases of Hardy type inequalities in weighted Lebesgue spaces [43, Section 1.3.2]. The proof of inequality (4.18) is subtler, and makes use of the fact that non-increasing trial functions $\varphi$ are considered. It follows via the same proof as (one of the cases) of [17, Theorem 5.1].

The super-critical regime, corresponding to the case when $p>n$, is the subject of the following theorem.

Theorem 4.4 [Super-critical Sobolev inequality] Let $\Omega$ be a open set in $\mathbb{R}^{n}, n \geq 2$, such that $\mathcal{L}^{n}(\Omega)<\infty$ and $\mathcal{H}^{n-1}(\partial \Omega)<\infty$. If $p>n$, then there exists a constant $C=$ $C\left(n, p, \mathcal{L}^{n}(\Omega), \mathcal{H}^{n-1}(\partial \Omega)\right)$ such that

$$
\|\mathbf{u}\|_{L^{\infty}(\Omega)} \leq C\left(\|\mathcal{E} \mathbf{u}\|_{L^{p}(\Omega)}+\|\mathbf{u}\|_{L^{\infty}(\partial \Omega)}\right)
$$

for every $u \in E^{1, p}(\Omega) \cap C_{b}(\bar{\Omega})$.

Proof of Theorem 4.4. Inequality 4.20 follows from Theorem 3.2 and Remark 3.3, via weighted Hardy type inequalities ([43, Section 1.3.2]).

The next result, contained in Theorem 4.5, concerns inequalities for functions whose symmetric gradient belongs to a Lorentz space $L^{p, q}(\Omega)$. It extends Theorems 4.1, 4.3 and 4.4, since $L^{p, p}(\Omega)=L^{p}(\Omega)$. In fact, the conclusion of Theorem 4.1 is also augmented by the result of Part (i) of Theorem 4.5, with che choice $q=p$. Actually, the Lorentz space $L^{\frac{\alpha p}{n-p}, p}(\Omega, \mu)$, which is obtained as a target space by Theorem 4.5 , is strictly contained in the Lebesgue space $L^{\frac{\alpha p}{n-p}}(\Omega, \mu)$ given by Theorem 4.1 .

Theorem 4.5 [Lorentz-Sobolev inequalities] Let $\Omega$ be any open set in $\mathbb{R}^{n}, n \geq 2$. Assume that $\mu$ is a measure in $\Omega$ fulfilling (3.1) for some exponent $\alpha \in(n-1, n]$ and for some constant $C_{\mu}$.

(i) Assume that $1<p<n$ and $1 \leq q \leq \infty$. Then there exists a constant $C=C\left(n, p, q, \alpha, C_{\mu}\right)$ such that

$$
\|\mathbf{u}\|_{L^{\frac{\alpha p}{n-p}, q}(\Omega, \mu)} \leq C\left(\|\mathcal{E} \mathbf{u}\|_{L^{p, q}(\Omega)}+\|\mathbf{u}\|_{L^{\frac{p(n-1)}{n-p}, q}(\partial \Omega)}\right)
$$


for every $u \in E^{1} L^{p, q}(\Omega) \cap C_{b}(\bar{\Omega})$.

(ii) Assume that $p=n$ and $q>1$, and that $\mathcal{L}^{n}(\Omega)<\infty, \mu(\Omega)<\infty$ and $\mathcal{H}^{n-1}(\partial \Omega)<\infty$. Then there exists a constant $C=C\left(n, q, \alpha, C_{\mu}, \mathcal{L}^{n}(\Omega), \mu(\Omega), \mathcal{H}^{n-1}(\partial \Omega)\right)$ such that

$$
\|\mathbf{u}\|_{L^{\infty ; q,-1}(\Omega, \mu)} \leq C\left(\|\mathcal{E} \mathbf{u}\|_{L^{n, q}(\Omega)}+\|\mathbf{u}\|_{L^{\infty ; q,-1}(\Omega)}\right)
$$

for every $u \in E^{1} L^{n, q}(\Omega) \cap C_{b}(\bar{\Omega})$.

(iii) Assume that either $p=n$ and $q=1$, or $p>n$ and $1 \leq q \leq \infty$, and that $\mathcal{L}^{n}(\Omega)<\infty, \mu(\Omega)<$ $\infty$ and $\mathcal{H}^{n-1}(\partial \Omega)<\infty$. Then there exists a constant $C=C\left(n, p, q, \alpha, C_{\mu}, \mathcal{L}^{n}(\Omega), \mu(\Omega), \mathcal{H}^{n-1}(\partial \Omega)\right)$ such that

$$
\|\mathbf{u}\|_{L^{\infty}(\Omega)} \leq C\left(\|\mathcal{E} \mathbf{u}\|_{L^{p, q}(\Omega)}+\|\mathbf{u}\|_{L^{\infty}(\partial \Omega)}\right)
$$

for every $u \in E^{1} L^{p, q}(\Omega) \cap C_{b}(\bar{\Omega})$.

Remark 4.6 Under the assumptions of Theorem 4.5, Part (ii), an inequality (slightly weaker than (4.22) involving Orlicz norms of exponential type can be shown to hold. It extends (4.8), and tells us that there exists a constant $C=C\left(n, q, \alpha, C_{\mu}, \mathcal{L}^{n}(\Omega), \mu(\Omega), \mathcal{H}^{n-1}(\partial \Omega)\right)$, such that

$$
\|\mathbf{u}\|_{\exp L^{\frac{q}{q-1}}(\Omega, \mu)} \leq C\left(\|\mathcal{E} \mathbf{u}\|_{L^{n, q}(\Omega)}+\|\mathbf{u}\|_{\exp L^{\frac{q}{q-1}}(\partial \Omega)}\right)
$$

for every $u \in E^{1} L^{n, q}(\Omega) \cap C_{b}(\bar{\Omega})$.

Proof of Theorem 4.5. Part (i). By the monotonicity of the function in 4.10), the couple of inequalities (3.11) and (3.12) in Theorem 3.2 applied with the norms appearing in inequality (4.21), is equivalent to the couple of inequalities

$$
\left\|s^{\frac{n-p}{\alpha p}-\frac{n-1}{\alpha}-\frac{1}{q}} \int_{0}^{s^{\frac{n}{\alpha}}} \varphi(r) d r\right\|_{L^{q}(0, \mu(\Omega))} \leq C\left\|s^{\frac{1}{p}-\frac{1}{q}} \varphi(s)\right\|_{L^{q}\left(0, \mathcal{L}^{n}(\Omega)\right)},
$$

and

$$
\left\|\int_{s^{\frac{n}{\alpha}}}^{\infty} r^{-\frac{n-1}{n}} \varphi(r) d r\right\|_{L^{\frac{\alpha p}{n-p}, q}(0, \mu(\Omega))} \leq C\|\varphi\|_{L^{p, q}\left(0, \mathcal{L}^{n}(\Omega)\right)}
$$

for some constant $C$ and for every non-increasing function $\varphi:(0, \infty) \rightarrow[0, \infty)$ with support in $\left(0, \mathcal{L}^{n}(\Omega)\right)$. Moreover, by the monotonicity of the function in 4.11 for any non-increasing function $\varphi$, inequality $(3.13)$ is equivalent to

$$
\left\|s^{\frac{n-p}{\alpha p}-\frac{n-1}{\alpha}-\frac{1}{q}} \int_{0}^{s^{\frac{n-1}{\alpha}}} \varphi(r) d r\right\|_{L^{q}(0, \mu(\Omega))} \leq C\left\|s^{\frac{n-p}{p(n-1)}-\frac{1}{q}} \varphi(s)\right\|_{L^{q}\left(0, \mathcal{H}^{n-1}(\partial \Omega)\right)},
$$

for some constant $C$ and for every non-increasing function $\varphi:(0, \infty) \rightarrow[0, \infty)$ with support in $\left(0, \mathcal{H}^{n-1}(\partial \Omega)\right)$. Inequalities (4.25) and (4.27) can be established via the criteria for weighted Hardy type inequalities in Lebesgue spaces [43, Section 1.3.2]. Inequality (4.26) follows from the proof of [17, Theorem 5.1]. With inequalities (4.25)-4.27) in place, inequality (4.21) is a consequence of Theorem 3.2 and Remark 3.3 .

Part (ii). The proof of inequality (4.22) follows along the same that of inequality (4.9). The details are omitted, for brevity.

Part (iii). Inequality (4.23) can be deduced from Theorem 3.2 and Remark 3.3, via weighted Hardy type inequalities in Lebesgue spaces [43, Section 1.3.2]. 
Our last result, contained in Theorem 4.7, consists of a set of inequalities for Orlicz norms of power-logarithmic type, i.e. Zugmund norms, of the symmetric gradient. They extend the results of Theorems 4.1, 4.3 and 4.4 in direction different from that of Theorem 4.5. It will be clear from the (sketched) proof that inequalities for more general Orlicz norms could be established via the same approach. Let us emphasize that the target norms are the strongest possible among all Orlicz spaces. Actually, they agree with the optimal Orlicz target norms appearing in parallel inequalities involving the full gradient. These inequalities are special cases of a result for arbitrary Orlicz-Sobolev spaces, established in [9, 10] in a classical setting, namely for the Lebesgue measure and for functions vanishing on the boundary, or defined on domains with some degree of regularity. The case of upper Ahlfors regular measures is considered in 18. Let us point out that an improvement of the conclusions of Theorem 4.7 is however still possible, if more general rearrangement-invariant target norms are allowed. The proof, like that of Theorem 4.7, relies upon Hardy type inequalities, with optimal rearrangement-invariant target spaces, from [14]. Orlicz-Sobolev inequalities, with optimal rearrangement-invariant norms, for the full gradient can be found in [11, 12] for the Lebesgue measure, and in [18] for upper Ahlfors regular measures. A discussion of these generalizations in the present framework is omitted, for brevity.

Theorem 4.7 [Zygmund-Sobolev inequalities] Let $\Omega$ be any open set in $\mathbb{R}^{n}, n \geq 2$. Let $\mu$ be a measure in $\Omega$ fulfilling (3.1) for some exponent $\alpha \in(n-1, n]$ and for some constant $C_{\mu}$. Assume that $\mathcal{L}^{n}(\Omega)<\infty, \mu(\Omega)<\infty$ and $\mathcal{H}^{n-1}(\partial \Omega)<\infty$.

(i) Assume that $1<p<n$ and $\sigma \in \mathbb{R}$. Then there exists a constant $C=C\left(n, p, \sigma, \alpha, C_{\mu}, \mathcal{L}^{n}(\Omega), \mu(\Omega), \mathcal{H}^{n-1}(\partial \Omega)\right)$ such that

$$
\|\mathbf{u}\|_{L^{\frac{p \alpha}{n-p}}(\log L)^{\frac{\sigma \alpha}{n-p}}(\Omega, \mu)} \leq C\left(\|\mathcal{E} \mathbf{u}\|_{L^{p}(\log L)^{\sigma}(\Omega)}+\|\mathbf{u}\|_{L^{\frac{p(n-1)}{n-p}}(\log L)^{\frac{\sigma(n-1)}{n-p}}(\partial \Omega)}\right)
$$

for every $u \in E^{1} L^{p}(\log L)^{\sigma}(\Omega) \cap C_{b}(\bar{\Omega})$.

(ii) Assume that $p=n$ and $\sigma<n-1$. Then there exists a constant $C=C\left(n, \sigma, \alpha, C_{\mu}, \mathcal{L}^{n}(\Omega), \mu(\Omega), \mathcal{H}^{n-1}(\partial \Omega)\right)$ such that

$$
\|\mathbf{u}\|_{\exp L^{\frac{n}{n-1-\sigma}(\Omega, \mu)}} \leq C\left(\|\mathcal{E} \mathbf{u}\|_{L^{n}(\log L)^{\sigma}(\Omega)}+\|\mathbf{u}\|_{\exp L^{\frac{n}{n-1-\sigma}}(\partial \Omega)}\right)
$$

for every $u \in E^{1} L^{n}(\log L)^{\sigma}(\Omega) \cap C_{b}(\bar{\Omega})$.

(iii) Assume that $p=n$ and $\sigma=n-1$. Then there exists a constant $C=C\left(n, \alpha, C_{\mu}, \mathcal{L}^{n}(\Omega), \mu(\Omega), \mathcal{H}^{n-1}(\partial \Omega)\right)$ such that

$$
\|\mathbf{u}\|_{\exp \exp L^{\frac{n}{n-1}(\Omega, \mu)}} \leq C\left(\|\mathcal{E} \mathbf{u}\|_{L^{n}(\log L)^{n-1}(\Omega)}+\|\mathbf{u}\|_{\exp \exp L^{\frac{n}{n-1}}(\partial \Omega)}\right)
$$

for every $u \in E^{1} L^{n}(\log L)^{n-1}(\Omega) \cap C_{b}(\bar{\Omega})$.

(iv) Assume that either $p=n$ and $\sigma>n-1$, or $p>n$ and $\sigma \in \mathbb{R}$. Then there exists a constant $C=C\left(n, p, \sigma, C_{\mu}, \mathcal{L}^{n}(\Omega), \mu(\Omega), \mathcal{H}^{n-1}(\partial \Omega)\right)$ such that

$$
\|\mathbf{u}\|_{L^{\infty}(\Omega)} \leq C\left(\|\mathcal{E} \mathbf{u}\|_{L^{p}(\log L)^{\sigma}(\Omega)}+\|\mathbf{u}\|_{L^{\infty}(\partial \Omega)}\right)
$$

$u \in E^{1} L^{p}(\log L)^{\sigma}(\Omega) \cap C_{b}(\bar{\Omega})$.

Proof, sketched. By Theorem 3.2 and Remark 3.3 , the proof is reduced to showing the validity of the inequalities:

$$
\left\|s^{-\frac{n-1}{\alpha}} \int_{0}^{s^{\frac{n}{\alpha}}} \varphi(r) d r\right\|_{L^{B}(0, \infty)} \leq C\|\varphi\|_{L^{A}(0, \infty)}
$$




$$
\left\|\int_{s^{\frac{n}{\alpha}}}^{\infty} r^{-\frac{n-1}{n}} \varphi(r) d r\right\|_{L^{B}(0, \infty)} \leq C\|\varphi\|_{L^{A}(0, \infty)}
$$

for some constant $C$ and for every non-increasing function $\varphi:(0, \infty) \rightarrow[0, \infty)$ with support in $\left(0, \mathcal{L}^{n}(\Omega)\right)$, and

$$
\left\|s^{-\frac{n-1}{\alpha}} \int_{0}^{s^{\frac{n-1}{\alpha}}} \varphi(r) d r\right\|_{L^{B}(0, \infty)} \leq C\|\varphi\|_{L^{D}(0, \infty)},
$$

for some constant $C$ and for every non-increasing function $\varphi:(0, \infty) \rightarrow[0, \infty)$ with support in $\left(0, \mathcal{H}^{n-1}(\partial \Omega)\right)$. Here, $A, B$, and $D$ are Young functions which yield the proper Orlicz spaces appearing in inequalities $(4.28)-(4.31)$. Notice that, owing to the assumption that $\mathcal{L}^{n}(\Omega)<\infty$, $\mu(\Omega)<\infty$ and $\mathcal{H}^{n-1}(\partial \Omega)<\infty$, only the behavior near infinity of the functions $A, B$, and $D$ is relevant here. Inequality $(4.33)$ is a special case of inequality (3.14) of [14, Theorem 3.5]. The same theorem can be exploited to deal with inequalities (4.32) and (4.34). Indeed, an Hölder type inequality in Orlicz spaces and Fubini's theorem ensure that

$$
\begin{aligned}
& \sup _{\varphi \in L^{A}(0, \infty)} \frac{\left\|s^{-\frac{n-1}{\alpha}} \int_{0}^{s^{\frac{n}{\alpha}}} \varphi(r) d r\right\|_{L^{B}(0, \infty)}}{\|\varphi\|_{L^{A}(0, \infty)}} \approx \sup _{\varphi \in L^{A}(0, \infty)} \sup _{\psi \in L^{\widetilde{B}}(0, \infty)} \frac{\int_{0}^{\infty} \psi(s) s^{-\frac{n-1}{\alpha}} \int_{0}^{s^{\frac{n}{\alpha}}} \varphi(r) d r d s}{\|\psi\|_{L^{\widetilde{B}}(0, \infty)}\|\varphi\|_{L^{A}(0, \infty)}} \\
& \approx \sup _{\psi \in L^{\widetilde{B}}(0, \infty)} \sup _{\varphi \in L^{A}(0, \infty)} \frac{\int_{0}^{\infty} \varphi(r) \int_{r^{\frac{\alpha}{n}}}^{\infty} \psi(s) s^{-\frac{n-1}{\alpha}} d s d r}{\|\psi\|_{L^{\widetilde{B}}(0, \infty)}\|\varphi\|_{L^{A}(0, \infty)}} \approx \sup _{\psi \in L^{\widetilde{B}}(0, \infty)} \frac{\left\|\int_{r^{\frac{\alpha}{n}}}^{\infty} \psi(s) s^{-\frac{n-1}{\alpha}} d s\right\|_{L^{\widetilde{A}}(0, \infty)}}{\|\psi\|_{L^{\widetilde{B}}(0, \infty)}} .
\end{aligned}
$$

Here, $\widetilde{A}$ and $\widetilde{B}$ denote the Young conjugates of $A$ and $B$, and the relation " $\approx$ " between two expressions means that they are bounded by each other, up to absolute multiplicative constants. Recall that $\widetilde{A}(t)=\sup \{s t-A(s): s \geq 0\}$ for $t \geq 0$. Therefore, inequality 4.32 is equivalent to

$$
\left\|\int_{r^{\frac{\alpha}{n}}}^{\infty} \psi(s) s^{-\frac{n-1}{\alpha}} d s\right\|_{L^{\widetilde{A}}(0, \infty)} \leq\|\psi\|_{L^{\widetilde{B}}(0, \infty)} .
$$

Similarly, inequality 4.34 is equivalent to

$$
\left\|\int_{r^{\frac{\alpha}{n-1}}}^{\infty} \psi(s) s^{-\frac{n-1}{\alpha}} d s\right\|_{L^{\tilde{D}}(0, \infty)} \leq\|\psi\|_{L^{\widetilde{B}}(0, \infty)} .
$$

Both inequalities (4.36) and (4.37) can be established via [14, Theorem 3.5].

\section{Compliance with Ethical Standards}

Funding. This research was partly funded by:

(i) Research Project of the Italian Ministry of University and Research (MIUR) Prin 2015 "Partial differential equations and related analytic-geometric inequalities" (grant number 2015HY8JCC);

(ii) GNAMPA of the Italian INdAM - National Institute of High Mathematics (grant number not available);

(iii) RUDN University Program 5-100.

Conflict of Interest. The authors declare that they have no conflict of interest. 


\section{References}

[1] E.Acerbi \& G.Mingione, Regularity results for stationary electro-rheological fluids, Arch. Rat. Mech. Anal. 164 (2002), 213-259.

[2] G. Astarita \& G. Marucci, Principles of non-Newtonian Fluid Mechanics. McGraw-Hill, London, 1974.

[3] C.Bennett \& R.Sharpley, Interpolation of operators, Academic Press, Boston, 1988.

[4] D.Breit, A.Cianchi \& L.Diening, Trace-free Korn inequalities in Orlicz spaces, SIAM J. Math. Anal. 49 (2017), 2496-2526.

[5] D.Breit \& L.Diening, Sharp conditions for Korn inequalities in Orlicz spaces, J. Math. Fluid Mech. 14 (2012), 565-573.

[6] D.Breit, L.Diening \& M. Fuchs, Solenoidal Lipschitz truncation and applications in fluid mechanics, J. Diff. Eq. 253 (2012), 1910-1942.

[7] M.Buliček, M.Majdoub, J.Málek, Unsteady flows of fluids with pressure dependent viscosity in unbounded domains, Nonlinear Anal. Real World Appl. 11 (2010), 3968-3983.

[8] S.Campanato, Proprietà di alcuni spazi di Banach connessi con la teoria dell'elasticità, Ann. Scuola Norm. Sup. Pisa 16 (1962), 121-142 (Italian).

[9] A.Cianchi, A sharp embedding theorem for Orlicz-Sobolev spaces, Indiana Univ. Math. J. 45 (1996), 39-65.

[10] A.Cianchi, Boundedness of solutions to variational problems under general growth conditions, Comm. Part. Diff. Equat. 22 (1997), 1629-1646.

[11] A.Cianchi, Optimal Orlicz-Sobolev embeddings, Rev. Mat. Iberoamericana 20 (2004), 427-474.

[12] A.Cianchi, Higher-order Sobolev and Poincaré inequalities in Orlicz spaces, Forum Math. 18 (2006), 745-767.

[13] A.Cianchi, Moser-Trudinger trace inequalities, Adv. Math. 217 (2008), 2005-2044.

[14] A.Cianchi, Orlicz-Sobolev boundary trace embeddings, Math. Z. 266 (2010), 431-449.

[15] A.Cianchi, Korn type inequalities in Orlicz spaces, J. Funct. Anal. 267 (2014), 2313-2352.

[16] A.Cianchi \& V.Maz'ya, Sobolev inequalities in arbitrary domains, Adv. Math. 293 (2016), 644696 .

[17] A.Cianchi \& L.Pick, Optimal Sobolev trace embeddings, Trans. Amer. Math. Soc. 368 (2016), 8349-8382.

[18] A.Cianchi, L.Pick \& L.Slavikova, Orlicz-Sobolev and Lorentz-Sobolev embeddings with measures, preprint.

[19] S.Conti, D.Faraco \& F.Maggi A new approach to Counterexamples to $L^{1}$ estimates: Korn's Inequality, geometric rigidity, and regularity for gradients of separately convex functions, Arch. Rat. Mech. Anal. 175 (2005), 287-300. 
[20] S.Dain, Generalized Korn's inequality and conformal Killing vectors, Calc. Var. Partial Differential Equations 25 (2006), 535-540.

[21] L.Diening, M.Ruzicka \& K.Schumacher, A decomposition technique for John domains, Ann. Acad. Scientiarum Fennicae 35 (2009), 87-114.

[22] E. Feireisl, Dynamics of Compressible Flow, Oxford University Press, Oxford, 2004.

[23] R.G.Durán \& M.A. Muschietti, The Korn inequality for Jones domains, Electron. J. Differential Equations 10 (2004), 10 pp. (electronic).

[DL] G.Duvaut \& J.L.Lions, Inequalities in Mechanics and Physics, Springer, Berlin, 1976.

[24] H. J. Eyring, Viscosity, plasticity, and diffusion as example of absolute reaction rates, J. Chemical Physics 4 (1936) 283-291.

[25] J.Frehse \& G.Seregin, Regularity of solutions to variational problems of the deformation theory of plasticity with logarithmic hardening, Proc. St. Petersburg Math. Soc. 5, 184222; English Translation: Amer. Math. Soc. Transl. II 193 (1998/1999), 127-152.

[26] M.Fuchs, On stationary incompressible Norton fluids and some extensions of Korn's inequality, Zeitschr. Anal. Anwendungen 13 (1994), 191-197.

[27] M.Fuchs, Korn inequalities in Orlicz spaces, Irish Math. Soc. Bull. 65 (2010), 5-9.

[28] M.Fuchs \& G.Seregin, Variational methods for problems from plasticity theory and for generalized Newtonian fluids, Lecture Notes in Mathematics Vol. 1749, Springer Verlag, Berlin-Heidelberg-New York, 2000.

[29] J.Gobert, Une inéquation fondamentale de la théorie de l'élasticité, Bull. Soc. Roy. Sci. Liege 3-4 (1962), 182-191.

[30] J.Gobert, Sur une inégalité de coercivité, J. Math. Anal. Appl. 36 (1971), 518-528.

[31] T.A.Hassan, V.K.Rangari \& S.Jeelani, Synthesis, processing and characterization of shear thickening fluid (STF) impregnated fabric composites, Materials Science and Engineering: A 527 (2010), 2892-2899.

[32] R.Kohn, New estimates for deformations in terms of their strains, PhD Thesis, Princetonn University, 1979.

[33] V.A.Kondratiev \& O.A.Oleinik, On Korn's inequalities, C. R. Acad. Sci. Paris Ser. I 308 (1989), 483-487.

[34] A.Korn, Über einige Ungleichungen, welche in der Theorie der elastischen und elektrischen Schwingungen eine Rolle spielen, in: Classe des Sciences Mathématiques et Naturels (9, Novembre), Bull. Internat. Acad. des Sci. Cracovie (1909), 705-724.

[35] K.de Leeuw \& H.Mirkil, A priori estimates for differential operators in $L_{\infty}$ norm, Illinois J. Math. 8 (1964), 112-124.

[36] P. L. Lions, Mathematical topics in fluid mechanics. Vol. 2. Compressible models. Oxford Science Publications, The Clarendon Press, Oxford University Press, New York, 1998. 
[37] F.Maggi \& C.Villani, Balls have the worst best Sobolev inequalities, J. Geom. Anal. 15 (2005), 83-121.

[38] F.Maggi \& C.Villani, Balls have the worst best Sobolev inequalities. II. Variants and extensions, Calc. Var. Partial Differential Equations 31 (2008), 47-74.

[39] J. Málek, J. Nečas, M. Rokyta \& M. Ružička, Weak and measure valued solutions to evolutionary PDEs, Chapman \& Hall, London, 1996.

[40] J.Málek \& K.R.Rajagopal, Mathematical issues concerning the Navier-Stokes equations and some of its generalizations, in Evolutionary equations, Vol. II, Handbook on Differential Equations, 371-459, C.Dafermos and E.Feireisl Eds., Elsevier/North-Holland, Amsterdam, 2005 .

[41] J. Málek \& K.R. Rajagopal, Compressible generalized Newtonian fluids, Zeit. Angew. Math. Physik (ZAMP) 61 (2010), 1097-1110.

[42] V.G.Maz'ya, Classes of regions and imbedding theorems for function spaces, Dokl. Akad. Nauk. SSSR 133 (1960), 527-530 (Russian); English translation: Soviet Math. Dokl. 1 (1960), 882-885.

[43] V.G.Maz'ya, Sobolev spaces with applications to elliptic partial differential equations, Springer, Heidelberg, 2011.

[44] P. P.Mosolov \& V. P.Mjasnikov, On the correctness of boundary value problems in the mechanics of continuous media, Math. USSR Sbornik 17 (1972), 257-267.

[45] P.Neff, D.Pauly \& K.-J.Witsch, Poincare meets Korn via Maxwell: extending Korn's first inequality to incompatible tensor fields, J. Diff. Equat. 258 (2015), 1267-1302.

[46] D.Ornstein, A non-inequality for differential operators in the $L_{1}$ norm, Arch. Rat. Mech. Anal. 11 (1964), 40-49.

[47] Yu.G.Reshetnyak, Estimates for certain differential operators with finite dimensional kernel, Sibirskii Math. Zh. 2 (1970), 414-418.

[48] A.Srivastavaa, A.Majumdara \& B.S.Butolaa, Improving the impact resistance of textile structures by using shear thickening fluids: a review, Critical Reviews in Solid State and Materials Sciences 37 (2012), 115-129.

[49] D.Spector \& J.Van Schaftingen, Optimal embeddings into Lorentz spaces for some vector differential operators via Gagliardo's lemma, preprint.

[50] M.Strauss, Variations of Korn's and Sobolev's inequalities, Proc. Symp. Pure Math. 23 D.Spencer Ed. , AMS, Providence R.I., 1973.

[51] R. Temam, Mathematical problems in plasticity, Gauthier Villars, Paris (1985).

[52] J.Van Schaftingen, J Limiting Sobolev inequalities for vector fields and canceling linear differential operators, J. Eur. Math. Soc. (JEMS) 15 (2013), 877-921.

[53] A.Wróblewska, Steady flow of non-Newtonian fluids-monotonicity methods in generalized Orlicz spaces, Nonlinear Anal. 72 (2010), 4136-4147. 